\title{
Synthesis of polyhydroquinoline derivatives via a four-component Hantzsch condensation catalyzed by tin dioxide nanoparticles
}

\author{
Seyed Mohammad VAHDAT a,*, Fereshteh CHEKIN a, Mehdi HATAMI ${ }^{b}$, \\ Maryam KHAVARPOUR c, Saeed BAGHERY d,e, Ziba ROSHAN-KOUHI e \\ a Department of Chemistry, Science and Research Ayatollah Amoli Branch, Islamic Azad University, Amol 678, Iran \\ ${ }^{\mathrm{b}}$ Marine Science Research Institute, Khoramshar University of Marine Science and Technology, Khoramshahr 64199-43175, Iran \\ c Chemical Engineering Department, Islamic Azad University, Ayatollah Amoli Branch, P.O. Box 678, Amol, Iran \\ ${ }^{d}$ Department of Chemistry, College of Science, Bu-Ali Sina University, Hamadan 65174, Iran \\ e Young Researchers Club, Science and Research Ayatollah Amoli Branch, Islamic Azad University, Amol 678, Iran
}

\section{A R T I C L E I N F O}

Article history:

Received 30 October 2012

Accepted 8 January 2013

Published 20 April 2013

\section{Keywords:}

Nano tin dioxide catalyst

Hantzsch condensation

Polyhydroquinoline

1,4-Dihydropyridine

\begin{abstract}
A B S T R A C T
Tin dioxide $\left(\mathrm{SnO}_{2}\right)$ nanoparticles efficiently catalyzed unsymmetrical four-component Hantzsch condensations of various aldehydes, dimedone, ethyl acetoacetate, and ammonium acetate to form polyhydroquinoline derivatives in excellent yields. This novel method offers several advantages over the traditional method of synthesizing these compounds, including safety, mild conditions, short reaction times, high yields, and an easy workup.
\end{abstract}

(C) 2013, Dalian Institute of Chemical Physics, Chinese Academy of Sciences. Published by Elsevier B.V. All rights reserved.

tion, and hydrothermal and polymeric precursor methods [7]. Because one-dimensional $\mathrm{SnO}_{2}$ structures (e.g., nanowires and nanorods) are expected to find wide applications in many fields, considerable effort has been directed at fabricating such structures by means of methods such as thermal oxidation and molten salt synthesis [8,9].

In this study, which is part of our ongoing investigations directed toward the development and improvement of new synthetic methodologies $[40,41]$, we investigated the use of $\mathrm{SnO}_{2}$ nanoparticles to catalyze the formation of polyhydroquinolines and 1,4-dihydropyridines (1,4-DHPs). In recent years, interest in the synthesis of 1,4-DHPs has increased, owing to their significant biological activities [10,11]. For example, various 1,4-DHPs with substituents at the 4-position have been reported to possess antiatherosclerotic, vasodilatory, antihypertensive, hepatoprotective, bronchodilatory, antimutagenic, 
antitumor, geroprotective, and antidiabetic activities [12-16]. DHPs have found commercial utility as calcium channel blockers (e.g., Nitrendipine, Nifedipine, and Nimodipine) [17]. DHPs can be synthesized by means of the Hantzsch condensation of an aldehyde, a $\beta$-ketoester, and ammonia; and replacement of ammonia by ammonium acetate permits the reaction to be carried out in aqueous media as well as under solvent-free conditions $[18,19]$.

Polyhydroquinolines, which are structurally related to DHPs, are another important group of nitrogen-containing heterocycles that have attracted much attention because of their diverse therapeutic and pharmacological properties, such as their ability to modulate calcium channels. Polyhydroquinolines have been synthesized under mild conditions augmented by conventional heating [20,21], microwave irradiation, and ultrasound [22,23]. In addition, various catalysts have been evaluated, including $\mathrm{FeF}_{3}$ [24], clay-supported $\mathrm{Ni}^{0}$ nanoparticles [25], unsupported nickel nanoparticles [26], silica-supported perchloric acid [27], titanium dioxide nanoparticles [28], Bakers' yeast [29], $\mathrm{K}_{7}\left[\mathrm{PW}_{11} \mathrm{CoO}_{40}\right.$ ] [30], scandium(III) triflate [31], ethylene glycol/MW [32], $p$-toluenesulfonic acid [33], trimethylsilyl chloride [34], palladium nanoparticles [35], a hafnium(IV) bis(perfluorooctanesulfonyl)imide complex [36], cerium(IV) ammonium nitrate [37], and iron(III) trifluoroacetate [38].

Despite the availability of these methods, the development of a mild and efficient catalyst for the synthesis of polyhydroquinolines remains highly desirable. In addition, the possibility of performing the reaction in aqueous media has attracted much attention because water is safer, cheaper, and more environmentally friendly than organic solvents [39]. A water-soluble catalyst that could be recycled by separation of the insoluble products by simple filtration is an especially attractive prospect.

Herein, we report a new, convenient, mild, efficient procedure for the synthesis of polyhydroquinoline derivatives by means of a $\mathrm{SnO}_{2}$ nanoparticle-catalyzed one-pot Hantzsch condensation of various aldehydes, dimedone, ethyl acetoacetate, and ammonium acetate at ambient temperature in ethanol.

\section{Experimental}

\subsection{General procedure for the Hantzsch synthesis of 1,4-DHPS}

A mixture of dimedone $(1.0 \mathrm{mmol})$, an aldehyde $(1.0 \mathrm{mmol})$, ethyl acetoacetate $(1.0 \mathrm{mmol})$, ammonium acetate $(1.0 \mathrm{mmol})$, and $\mathrm{SnO}_{2}$ nanoparticles ( $\left.1 \mathrm{~mol} \%\right)$ in ethanol ( $\left.2 \mathrm{ml}\right)$ was stirred at room temperature until the reaction was complete, as indicated by thin-layer chromatography ( $n$-hexane/ethyl acetate 5:1). The crude solid product was filtered and then purified by recrystallization from ethanol-water. The physical data (mp, NMR, IR) of known compounds were identical to the corresponding literature data.

\subsection{General procedure for the synthesis of polyhydroquinolines}

A mixture of dimedone $(1.0 \mathrm{mmol})$, aldehyde $(1.0 \mathrm{mmol})$, ethyl acetoacetate $(1.0 \mathrm{mmol})$, ammonium acetate $(1.0 \mathrm{mmol})$ and nano $\mathrm{SnO}_{2}$ catalyst ( $\left.1 \mathrm{~mol} \%\right)$ in ethanol $(2 \mathrm{ml})$ were stirred at room temperature for an appropriate time. The progress of the reaction was monitored by TLC ( $n$-hexan/ethyl acetate 5:1). After completion of the reaction, the resulting solid (crude product) was filtered and then recrystallized from ethanol-water to obtain pure product. The physical data (mp, NMR, IR) of these known compounds were found to be identical with those reported in the literature.

Ethyl 4-(4-chlorophenyl)-2,7,7-trimethyl-5-oxo-1,4,5,6,7,8hexahydroquinoline-3-carboxylate (Table 4 entry 3) Yield: 96\%; mp 229-231 ${ }^{\circ} \mathrm{C}$ (lit. [46] 230-232 ${ }^{\circ} \mathrm{C}$ ). ${ }^{1} \mathrm{H}$ NMR (400 MHz, DMSO): $\delta 0.84\left(\mathrm{~s}, 3 \mathrm{H},-\mathrm{CH}_{3}\right), 0.99\left(\mathrm{~s}, 3 \mathrm{H},-\mathrm{CH}_{3}\right), 1.14(\mathrm{t}, 3 \mathrm{H}, J=$ $7.2 \mathrm{~Hz},-\mathrm{CH}_{3}$ ), 2.06 (dd, $2 \mathrm{H},-\mathrm{CH}_{2}-$ ), 2.13 (dd, $2 \mathrm{H},-\mathrm{CH}_{2}-$ ), 2.28 (s, $\left.3 \mathrm{H},-\mathrm{CH}_{3}\right), 3.99$ (q, 2H, J= $7.3 \mathrm{~Hz},-\mathrm{OCH}_{2}-$ ), 4.95 (s, 1H, -CH-), 7.08 (d, 2H, $J=9.3 \mathrm{~Hz}, \mathrm{Ar}-\mathrm{H}$ ), 7.18 (d, 2H, $J=9.4 \mathrm{~Hz}, \mathrm{Ar}-\mathrm{H}$ ), 7.93 (brs, $1 \mathrm{H},-\mathrm{NH}-$ ). ${ }^{13} \mathrm{C}$ NMR (100 MHz, DMSO): $\delta 14.8,19.2,27.4$, 29.9, 32.8, 36.6, 40.8, 51.2, 60.1, 77.8, 105.3, 111.5, 128.2, 129.8, 131.7, 146.5, 150.1, 167.8, 195.7; IR (KBr, cm-1) 3275, 3199, 3073, 2931, 2360, 1676, 1604, 1490, 1380, 1214, 1101, 857.

Ethyl 4-(4-hydroxy-3-methoxyphenyl)-2,7,7-trimethyl-5oxo-1,4,5,6,7,8-hexahydroquinoline-3-carboxylate (Table 4 entry 13). Yield: $96 \%$; mp $212-214^{\circ} \mathrm{C}$ (lit. [48] 210-212 ${ }^{\circ} \mathrm{C}$ ); ${ }^{1 \mathrm{H}}$ NMR (400 MHz, DMSO): $\delta 0.93\left(\mathrm{~s}, 3 \mathrm{H},-\mathrm{CH}_{3}\right), 1.04\left(\mathrm{~s}, 3 \mathrm{H},-\mathrm{CH}_{3}\right)$, $1.25\left(\mathrm{t}, 3 \mathrm{H}, J=7.2 \mathrm{~Hz},-\mathrm{CH}_{3}\right), 1.91-2.18\left(\mathrm{dd}, 2 \mathrm{H},-\mathrm{CH}_{2}-\right.$ ), 2.27-2.39 (dd, 2H, $-\mathrm{CH}_{2}-$ ) 2.27 (s, 3H, $\left.-\mathrm{CH}_{3}\right), 3.73$ (s, 3H, $\left.-\mathrm{OCH}_{3}\right), 4.06\left(\mathrm{q}, 2 \mathrm{H}, \mathrm{J}=7.3 \mathrm{~Hz},-\mathrm{OCH}_{2}-\right), 4.71(\mathrm{~s}, 1 \mathrm{H},-\mathrm{CH}-)$, 6.49-6.77 (m, 3H, Ar-H), 8.07 (brs, 1H, -OH), 8.58 (brs, 1H, -NH-). ${ }^{13}$ C NMR (100 MHz, DMSO): $\delta 15.3,27.1,28.8,33.1,38.4$, 47.6, 52.4, 58.3, 106.7, 109.1, 111.3, 115.1, 121.5, 137.2, 145.6, 147.4, 153.3, 157.1, 167.3, 197.6; IR (KBr, cm-1) 3397, 3285, $3065,2919,1688,1559,1471,1267,1223,829$.

Ethyl 2,7,7-trimethyl-5-oxo-4-p-tolyl-1,4,5,6,7,8-hexahydroquinoline-3-carboxylate (Table 4 entry 15). Yield: 96\%; mp 263-265 ${ }^{\circ} \mathrm{C}$ (lit. [45] 260-261 ${ }^{\circ} \mathrm{C}$ ). ${ }^{1} \mathrm{H}$ NMR (400 MHz, DMSO): $\delta$ $0.81\left(\mathrm{~s}, 3 \mathrm{H},-\mathrm{CH}_{3}\right), 1.07\left(\mathrm{~s}, 3 \mathrm{H},-\mathrm{CH}_{3}\right), 1.18(\mathrm{t}, 3 \mathrm{H}, J=7.2 \mathrm{~Hz}$, $-\mathrm{CH}_{3}$ ), $1.98\left(\mathrm{~s}, 3 \mathrm{H},-\mathrm{CH}_{3}\right), 2.03$ (dd, $2 \mathrm{H},-\mathrm{CH}_{2}-$ ), 2.21 (dd, $2 \mathrm{H}$, $\left.-\mathrm{CH}_{2}-\right), 2.41\left(\mathrm{~s}, 3 \mathrm{H},-\mathrm{CH}_{3}\right), 4.06\left(\mathrm{q}, 2 \mathrm{H}, J=7.1 \mathrm{~Hz},-\mathrm{OCH}_{2}-\right), 5.11$ (s, 1H, -CH-), 7.44 (d, 2H, J = 9.2 Hz, Ar-H), 8.15 (d, 2H, $J=9.2$ $\mathrm{Hz}, \mathrm{Ar}-\mathrm{H}$ ), 8.21 (brs, 1H, -NH-). ${ }^{13} \mathrm{C}$ NMR (100 MHz, DMSO): $\delta$ 14.3, 18.9, 23.1, 27.1, 28.5, 31.9, 37.6, 40.7, 51.3, 61.9, 77.7, 104.3, 110.9, 122.8, 129.1, 146.5, 150.3, 155.1, 168.1, 197.1; IR $\left(\mathrm{KBr}, \mathrm{cm}^{-1}\right)$ 3270, 3185, 3075, 2967, 2366, 1669, 1614, 1567, 1490, 1372, 1276, 1151, 875.

Ethyl 2,7,7-trimethyl-4-(4-nitrophenyl)-5-oxo-1,4,5,6,7,8hexahydroquinoline-3-carboxylate (Table 4 entry 20). Yield: 98\%; mp 244-246 ${ }^{\circ} \mathrm{C}$ (lit. [25] 242-244 ${ }^{\circ} \mathrm{C}$ ). ${ }^{1} \mathrm{H}$ NMR (400 MHz, DMSO): $\delta 0.84\left(\mathrm{~s}, 3 \mathrm{H},-\mathrm{CH}_{3}\right), 1.01\left(\mathrm{~s}, 3 \mathrm{H},-\mathrm{CH}_{3}\right), 1.13(\mathrm{t}, 3 \mathrm{H}, J=$ $7.2 \mathrm{~Hz},-\mathrm{CH}_{3}$ ), 2.01 (dd, $2 \mathrm{H},-\mathrm{CH}_{2}-$ ), 2.15 (dd, $2 \mathrm{H},-\mathrm{CH}_{2}-$ ), 2.32 (s, 3H, $\left.-\mathrm{CH}_{3}\right), 4.01$ (q, 2H, J= $7.3 \mathrm{~Hz},-\mathrm{OCH}_{2}-$ ), 5.10 (s, 1H, -CH-), 7.44 (d, 2H, $J=9.3 \mathrm{~Hz}, \mathrm{Ar}-\mathrm{H}$ ), 8.01 (d, 2H, $J=9.4 \mathrm{~Hz}, \mathrm{Ar}-\mathrm{H}$ ), 8.12 (brs, $1 \mathrm{H},-\mathrm{NH}-) .{ }^{13} \mathrm{C}$ NMR (100 MHz, DMSO): $\delta 14.6,19.3,27.4$, 29.8, 32.9, 37.6, 40.9, 51.1, 60.2, 77.9, 104.5, 110.8, 123.6, 129.3, 146.4, 150.3, 155.3, 167.5, 195.8; IR (KBr, cm-1) 3276, 3189, 3072, 2967, 2360, 1679, 1604, 1557, 1490, 1342, 1276, 1105, 879. 
Table 1

Effect of catalyst amount on the yield of the Hantzsch condensation reaction.

\begin{tabular}{lc}
\hline Amount of catalyst (mol\%) & Isolated yield (\%) \\
\hline 0.5 & 89 \\
1 & 94 \\
2 & 93 \\
5 & 89 \\
10 & 87 \\
\hline
\end{tabular}

Reaction conditions: benzaldehyde $(1 \mathrm{mmol})$, dimedone $(1 \mathrm{mmol})$, ethyl acetoacetate $(1 \mathrm{mmol})$, ammonium acetate $(1 \mathrm{mmol})$, ethanol volume ( $2 \mathrm{ml})$, room temperature.

Diethyl 4-(4-chlorophenyl)-2,6-dimethyl-1,4-dihydropyridine-3,5-dicarboxylate (Table 6 entry 2). Yield: 95\%; mp 145-147 ${ }^{\circ} \mathrm{C}$ (lit. [53] 144-146 ${ }^{\circ} \mathrm{C}$ ). ${ }^{1} \mathrm{H}$ NMR (400 MHz, DMSO): $\delta$ $1.23\left(\mathrm{t}, 6 \mathrm{H}, \mathrm{J}=7.1 \mathrm{~Hz},-\mathrm{CH}_{3}\right), 2.27\left(\mathrm{~s}, 6 \mathrm{H},-\mathrm{CH}_{3}\right), 4.17(\mathrm{~m}, 4 \mathrm{H}, \mathrm{J}=$ $5.7 \mathrm{~Hz},-\mathrm{OCH}_{2}$ ), 4.87 (brs, 1H, -NH-), 5.68 (s, 1H, -CH-), 7.41 (d, $2 \mathrm{H}, J=7.9 \mathrm{~Hz}, \mathrm{Ar}-\mathrm{H}), 7.57$ (d, 2H, J= $7.8 \mathrm{~Hz}, \mathrm{Ar}-\mathrm{H}) .{ }^{13} \mathrm{C}$ NMR $(100$ MHz, DMSO): $\delta 14.3,14.9,45.9,61.3,97.6,127.1,128.3,134.8$, 143.2, 147.1, 167.8; IR (KBr, cm-1) 3273, 3180, 2967, 1687, $1557,1495,1341,1267,879$.

Diethyl 2,6-dimethyl-4-(4-nitrophenyl)-1,4-dihydropyridine-3,5-dicarboxylate (Table 6 entry 7): Yield: 98\%; mp 131-133 ${ }^{\circ} \mathrm{C}$ (lit. [53] 130-132 ${ }^{\circ} \mathrm{C}$ ). ${ }^{1} \mathrm{H}$ NMR (400 MHz, DMSO): $\delta$ $1.27\left(\mathrm{t}, 6 \mathrm{H}, J=7.3 \mathrm{~Hz},-\mathrm{CH}_{3}\right), 2.13\left(\mathrm{~s}, 6 \mathrm{H},-\mathrm{CH}_{3}\right), 4.35(\mathrm{~m}, 4 \mathrm{H}, J=$ $5.9 \mathrm{~Hz},-\mathrm{OCH}_{2}$ ), 4.85 (brs, $1 \mathrm{H},-\mathrm{NH}-$ ), 5.79 (s, 1H, -CH-), 7.51 (d, $2 \mathrm{H}, J=7.8 \mathrm{~Hz}, \mathrm{Ar}-\mathrm{H}), 7.59$ (d, 2H, J= $7.8 \mathrm{~Hz}, \mathrm{Ar}-\mathrm{H}) ;{ }^{13} \mathrm{C}$ NMR $(100$ MHz, DMSO): $\delta$ 15.1, 17.8, 45.7, 63.3, 96.6, 128.1, 128.7, 132.5, 145.2, 148.3, 167.5; IR (KBr, cm-1) 3275, 3191, 2975, 1657, $15357,1491,1356,1265,875$.

\section{Results and discussion}

First, we optimized the reaction conditions for the four-component Hantzsch condensation with respect to yield and reaction rate by varying the catalyst amount (Table 1), the solvent (Table 2), and the reaction temperature. We obtained the maximum yield of the desired polyhydroquinolines (94\%) with $1 \mathrm{~mol} \%$ of catalyst at room temperature in ethanol, and
Table 2

Effect of solvent on the reaction time and yield of the Hantzsch condensation reaction.

\begin{tabular}{lcc}
\hline Solvent & Time (min) & Isolated yield (\%) \\
\hline $\mathrm{H}_{2} \mathrm{O}$ & 23 & 71 \\
$\mathrm{C}_{2} \mathrm{H}_{5} \mathrm{OH}$ & 9 & 94 \\
$\mathrm{CH}_{3} \mathrm{CN}$ & 12 & 93 \\
$\mathrm{THF}$ & 15 & 91 \\
Toluene & 19 & 89 \\
\hline
\end{tabular}

Reaction conditions: benzaldehyde $(1 \mathrm{mmol})$, dimedone $(1 \mathrm{mmol})$, ethyl acetoacetate $(1 \mathrm{mmol})$, ammonium acetate $(1 \mathrm{mmol}), \mathrm{SnO}_{2}$ nanoparticles ( $1 \mathrm{~mol} \%)$, solvent volume $(2 \mathrm{ml})$, room temperature.

under these conditions, the reaction was complete within 9 min (Scheme 1).

We compared the results we obtained with $\mathrm{SnO}_{2}$ nanoparticles as a catalyst with the results reported in the literature for various other catalysts in similar reactions (Table 3). Two of the literature methods required a catalyst ratio of $>1 \mathrm{~mol} \%$ (entries 4 and 7), and some of the methods required much longer reaction times (entries 2, 7, and 8).

We next evaluated the substrate scope of the reaction with a series of aromatic, aliphatic, and heterocyclic aldehydes and found that all the tested substrates underwent the electrophilic substitution reaction to produce a wide range of polyhydroquinoline derivatives in good to excellent yields (Table 4). Aromatic aldehydes with electron-withdrawing groups on the aromatic ring (entries 18-21) reacted faster than aldehydes bearing electron-donating groups (entries 10, 12, and 15). Aliphatic aldehydes also required relatively long reaction times (entries 25-28), most likely because of the electron-donating and steric effects of the aliphatic moieties.

We evaluated the reusability of the catalysts by carrying out multiple reactions of dimedone, 4-nitrobenzaldehyde, ethyl acetoacetate, and ammonium acetate to form 4at (Table 5). After completion of each reaction, the product was extracted with ethanol, and the catalyst was recovered from the aqueous layer. The $\mathrm{SnO}_{2}$ nanoparticles were more soluble in ethanol than in water. In this medium, the recovered catalyst could be reused five times without appreciable loss of catalytic activity.

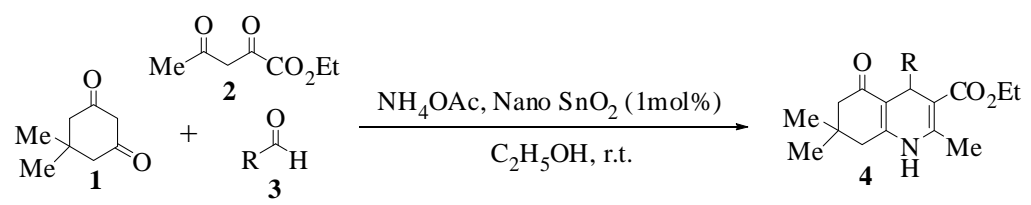

Scheme 1. $\mathrm{SnO}_{2}$ nanoparticle-catalyzed synthesis of polyhydroquinolines 4 .

Table 3

Comparison of various catalysts in the four-component Hantzsch condensation.

\begin{tabular}{|c|c|c|c|c|c|c|}
\hline Entry & Catalyst (mol\%) & Solvent & Temperature $\left({ }^{\circ} \mathrm{C}\right)$ & Time (min) & Yield (\%) & Ref. \\
\hline 1 & $\mathrm{SnO}_{2} / 1$ & $\mathrm{C}_{2} \mathrm{H}_{5} \mathrm{OH}$ & r.t & 9 & 94 & this work \\
\hline 2 & Bakers' yeast-D-glucose/200-300 mg & phosphate buffer $(\mathrm{pH}=7.0)$ & r.t & 1440 & 79 & [29] \\
\hline 3 & MCM-41/1 & solvent free & 90 & 15 & 89 & [38] \\
\hline 4 & $\mathrm{ZnO} / 10$ & $\mathrm{C}_{2} \mathrm{H}_{5} \mathrm{OH}$ & 80 & 60 & 92 & {$[42]$} \\
\hline 5 & SBA-Pr- $\mathrm{SO}_{3} \mathrm{H} / 0.05 \mathrm{~g}$ & solvent free & 80 & 10 & 85 & {$[43]$} \\
\hline 6 & $\mathrm{~K}_{7}\left[\mathrm{PW}_{12} \mathrm{CoO}_{40}\right] / 1$ & $\mathrm{CH}_{3} \mathrm{CN} /$ reflux & - & 35 & 80 & {$[30]$} \\
\hline 7 & L-proline/10 & $\mathrm{C}_{2} \mathrm{H}_{5} \mathrm{OH} /$ reflux & - & 360 & 92 & {$[44]$} \\
\hline 8 & $\mathrm{Hf}\left(\mathrm{NPf}_{2}\right)_{4} / 1$ & perfluorodecalin $\left(\mathrm{C}_{10} \mathrm{~F}_{18}\right)$ & 60 & 180 & 95 & [36] \\
\hline
\end{tabular}


Table 4

$\mathrm{SnO}_{2}$ nanoparticle-catalyzed Hantzsch synthesis of polyhydroquinoline derivatives from various aldehydes (RCHO).

\begin{tabular}{|c|c|c|c|c|c|c|}
\hline Entry & $\mathrm{R}$ & Product & $\mathrm{mp}\left({ }^{\circ} \mathrm{C}\right)$ & Reaction time (min) & Yield (\%) & Ref. \\
\hline 1 & $\mathrm{C}_{6} \mathrm{H}_{5}$ & 4aa & $203-205$ & 9 & 94 & {$[45]$} \\
\hline 2 & $2-\mathrm{Cl}-\mathrm{C}_{6} \mathrm{H}_{4}$ & 4ab & $209-211$ & 7 & 95 & {$[34]$} \\
\hline 3 & $4-\mathrm{Cl}-\mathrm{C}_{6} \mathrm{H}_{4}$ & $4 a c$ & $229-231$ & 6 & 96 & {$[46]$} \\
\hline 4 & $2,4-\mathrm{Cl}_{2}-\mathrm{C}_{6} \mathrm{H}_{3}$ & 4ad & $240-242$ & 6 & 96 & [29] \\
\hline 5 & $3,4-\mathrm{Cl}_{2}-\mathrm{C}_{6} \mathrm{H}_{3}$ & 4ae & $200-202$ & 7 & 95 & {$[47]$} \\
\hline 6 & $2,6-\mathrm{Cl}_{2}-\mathrm{C}_{6} \mathrm{H}_{3}$ & 4af & $243-245$ & 6 & 95 & [29] \\
\hline 7 & $4-\mathrm{F}-\mathrm{C}_{6} \mathrm{H}_{4}$ & 4ag & $185-187$ & 6 & 96 & [37] \\
\hline 8 & $4-\mathrm{Br}-\mathrm{C}_{6} \mathrm{H}_{4}$ & $4 a h$ & $253-255$ & 6 & 95 & {$[45]$} \\
\hline 9 & $3-\mathrm{OH}-\mathrm{C}_{6} \mathrm{H}_{4}$ & 4ai & $216-218$ & 9 & 94 & {$[26]$} \\
\hline 10 & $4-\mathrm{OH}-\mathrm{C}_{6} \mathrm{H}_{4}$ & 4aj & $228-230$ & 8 & 95 & {$[45]$} \\
\hline 11 & $3-\mathrm{OMe}-\mathrm{C}_{6} \mathrm{H}_{4}$ & 4ak & $201-203$ & 8 & 94 & {$[34]$} \\
\hline 12 & 4-OMe- $\mathrm{C}_{6} \mathrm{H}_{4}$ & 4al & $258-260$ & 7 & 95 & {$[45]$} \\
\hline 13 & 4-OH-3-OMe- $\mathrm{C}_{6} \mathrm{H}_{3}$ & 4am & $212-214$ & 8 & 96 & {$[48]$} \\
\hline 14 & $3,4,5-(\mathrm{OMe})_{3}-\mathrm{C}_{6} \mathrm{H}_{2}$ & 4an & 197-199 & 8 & 95 & {$[20]$} \\
\hline 15 & 4-Me- $\mathrm{C}_{6} \mathrm{H}_{4}$ & 4 ao & $263-265$ & 6 & 96 & {$[45]$} \\
\hline 16 & $4-(\mathrm{Me})_{2} \mathrm{CH}-\mathrm{C}_{6} \mathrm{H}_{4}$ & 4ap & $181-183$ & 7 & 96 & {$[49]$} \\
\hline 17 & $4-(\mathrm{Me})_{2} \mathrm{~N}-\mathrm{C}_{6} \mathrm{H}_{4}$ & $4 a q$ & $231-233$ & 5 & 97 & {$[27]$} \\
\hline 18 & $4-\mathrm{CF}_{3}-\mathrm{C}_{6} \mathrm{H}_{4}$ & 4ar & 190-192 & 2 & 98 & {$[24]$} \\
\hline 19 & $3-\mathrm{NO}_{2}-\mathrm{C}_{6} \mathrm{H}_{4}$ & 4as & $175-177$ & 3 & 97 & {$[20]$} \\
\hline 20 & $4-\mathrm{NO}_{2}-\mathrm{C}_{6} \mathrm{H}_{4}$ & 4at & $244-246$ & 1 & 98 & {$[25]$} \\
\hline 21 & $4-\mathrm{CN}-\mathrm{C}_{6} \mathrm{H}_{4}$ & $4 a u$ & $143-145$ & 2 & 97 & {$[24]$} \\
\hline 22 & cinnamaldehyde & 4av & $203-205$ & 10 & 94 & {$[31]$} \\
\hline 23 & furan-2-carbaldehyde & 4aw & $246-248$ & 9 & 95 & {$[50]$} \\
\hline 24 & thiophene-2-carbaldehyde & $4 a x$ & $225-227$ & 10 & 95 & {$[34]$} \\
\hline 25 & $\mathrm{CH}_{3} \mathrm{CH}_{2}$ & $4 \mathrm{ba}$ & $143-145$ & 15 & 91 & {$[45]$} \\
\hline 26 & $\mathrm{CH}_{3}\left(\mathrm{CH}_{2}\right)_{2}$ & $4 b b$ & $148-150$ & 14 & 92 & [45] \\
\hline 27 & $\left(\mathrm{CH}_{3}\right)_{2} \mathrm{CH}$ & $4 b c$ & $161-163$ & 15 & 92 & {$[44]$} \\
\hline 28 & $\mathrm{CH}_{3}\left(\mathrm{CH}_{2}\right)_{4}$ & 4bd & $165-167$ & 15 & 91 & [51] \\
\hline
\end{tabular}

Table 5

Evaluation of catalyst reusability for the synthesis of 4at.

\begin{tabular}{lcc}
\hline Entry & Isolated yield (\%) & Catalyst recovery (\%) \\
\hline 1 & 98 & 98 \\
2 & 97 & 96 \\
3 & 97 & 95 \\
4 & 96 & 93 \\
5 & 95 & 90 \\
\hline
\end{tabular}

The morphologies of fresh $\mathrm{SnO}_{2}$ nanoparticles, a composite sample containing $\mathrm{SnO}_{2}$ nanoparticles and product, and $\mathrm{SnO}_{2}$ nanoparticles recovered after three reactions were studied by field emission scanning electron microscopy (Fig. 1). The images of the fresh nanoparticles showed spherical grains with diameters of 30-60 nm with condensed structure and no aggregation (Fig. 1(a)). The image of the composite sample
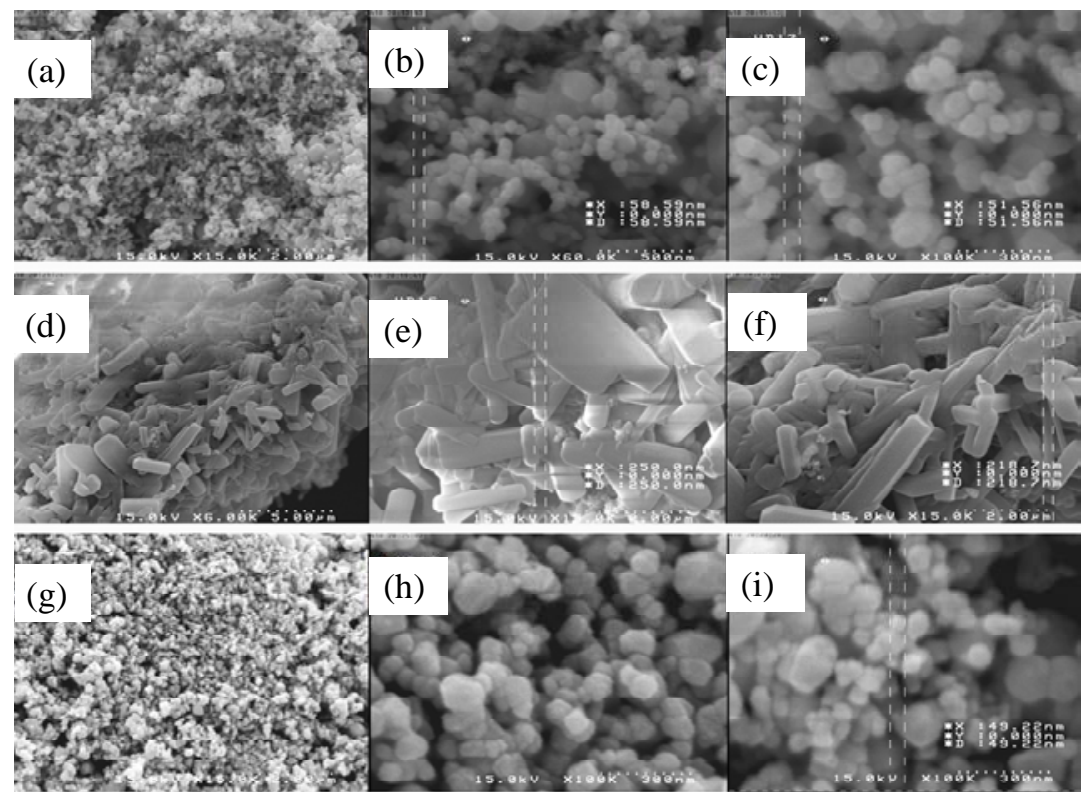

Fig. 1. Field emission scanning electron microscopy images of fresh $\mathrm{SnO}_{2}$ nanoparticles (a-c), a composite sample containing $\mathrm{SnO}_{2}$ nanoparticles and reaction product (d-f), and $\mathrm{SnO}_{2}$ nanoparticles recovered after three uses (g-i). 


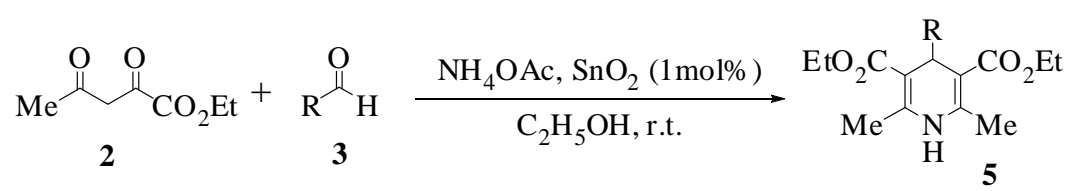

Scheme 2. $\mathrm{SnO}_{2}$ nanoparticle-catalyzed synthesis of 1,4-DHPs.

Table 6

$\mathrm{SnO}_{2}$ nanoparticle-catalyzed three-component Hantzsch synthesis of 1,4-DHPs from various aldehydes (RCHO).

\begin{tabular}{|c|c|c|c|c|c|c|}
\hline Entry & $\mathrm{R}$ & Product & $\mathrm{mp}\left({ }^{\circ} \mathrm{C}\right)$ & Time (min) & Yield (\%) & Ref. \\
\hline 1 & $\mathrm{Ph}$ & $\mathbf{5 a}$ & $158-160$ & 11 & 93 & {$[52]$} \\
\hline 2 & $4-\mathrm{Cl}_{-} \mathrm{C}_{6} \mathrm{H}_{5}$ & $\mathbf{5 b}$ & $145-147$ & 7 & 95 & [53] \\
\hline 3 & $4-\mathrm{OH}-\mathrm{C}_{6} \mathrm{H}_{5}$ & $5 c$ & $231-233$ & 9 & 94 & [53] \\
\hline 4 & $4-\mathrm{OMe}-\mathrm{C}_{6} \mathrm{H}_{5}$ & $5 d$ & $151-153$ & 7 & 94 & [52] \\
\hline 5 & 4-Me- $\mathrm{C}_{6} \mathrm{H}_{5}$ & $5 e$ & $137-139$ & 8 & 95 & [54] \\
\hline 6 & $3-\mathrm{NO}_{2}-\mathrm{C}_{6} \mathrm{H}_{5}$ & $5 f$ & $166-168$ & 5 & 97 & [54] \\
\hline 7 & $4-\mathrm{NO}_{2}-\mathrm{C}_{6} \mathrm{H}_{5}$ & $5 g$ & $131-133$ & 2 & 98 & [53] \\
\hline 8 & cinnamaldehyde & $5 \mathrm{~h}$ & $120-122$ & 14 & 93 & [53] \\
\hline 9 & furan-2-carbaldehyde & $5 \mathbf{i}$ & $161-163$ & 11 & 93 & [52] \\
\hline
\end{tabular}

showed the presence of cubic rectangular structures with lengths of $>200 \mathrm{~nm}$ for reaction products and the presence of nanoparticles in the solution. The images of the nanoparticles recovered from the reaction medium revealed that some aggregates were present.

We also evaluated the use of $\mathrm{SnO}_{2}$ nanoparticle-catalyzed three-component Hantzsch reactions for the efficient synthesis of 1,4-DHPs at room temperature (Scheme 2). The condensation of $2 \mathrm{mmol}$ of ethyl acetoacetate with $1 \mathrm{mmol}$ of each of various arylaldehydes and $\mathrm{NH}_{4} \mathrm{OAc}$ in ethanol in the presence of $\mathrm{SnO}_{2}$ nanoparticles rapidly furnished the desired 1,4-DHPs in high yields (Scheme 2).

Evaluation of the substrate scope of the reaction revealed that aromatic aldehydes with electron-withdrawing groups on the aromatic ring (Table 6, entries 6 and 7) reacted faster than aldehydes bearing electron-donating groups (entries 3-5).

\section{Conclusions}

$\mathrm{SnO}_{2}$ nanoparticles efficiently catalyzed the Hantzsch condensation of various aldehydes, dimedone, ethyl acetoacetate, and ammonium acetate in ethanol to produce polyhydroquinolines in excellent yields. The catalyst offered several advantages over previously reported catalysts, including fewer byproducts, mild reaction conditions, high yields, shorter reaction times, and lower catalyst loads; in addition, the reactions were simple to carry out, and the products were easy to isolate. The nanoparticle catalyst could be reused at least five times with only minimal reduction in catalytic activity of the recovered catalyst. These advantages of this catalyst make this method a benign, economical, and waste-free process for the synthesis of polyhydroquinolines.

\section{Acknowledgments}

The authors are sincerely thankful for the research facilities provided by the Ayatollah Amoli Branch of the Islamic Azad University.

\section{References}

[1] Zhu J, Tay B Y, Ma J. Mater Lett, 2006, 60: 1003

[2] Nayral C, Viala E, Colliere V, Fau P, Senocq F, Maisonnat A, Chaudret B. Appl Surf Sci, 2000, 164: 219

[3] Fort A, Mugnaini M, Rocchi S, Serrano-Santos M B, Vignoli V, Spinicci R. Sens Actuators B, 2007, 124: 245

[4] Pyke D R, Reid R T, Tilley R J D. J Chem Soc, Faraday Trans 1, 1980, 76: 1174

[5] Kawabe T, Tabata K, Suzuki E, Ichikawa Y, Nagasawa Y. Catal Today, 2001, 71: 21

[6] Chi K M, Lin C C, Lu Y H, Liao J H. J Chin Chem Soc, 2000, 47: 425

[7] Ibarguen A C, Mosquera A, Parra R, Castro M S, Rodríguez-Páez J E. Mater Chem Phys, 2007, 101: 433

[8] Zheng M J, Li G H, Zhang X Y, Huang S Y, Lei Y, Zhang L D. Chem Mater, 2001, 13: 3859

[9] Liu Y K, Zheng, C L, Wang W Z, Yin C R, Wang G H. Adv Mater, 2001, 13: 1883

[10] Shan R, Velazquez C, Knaus E E. J Med Chem, 2004, 47: 254

[11] Sawada Y, Kayakiri H, Abe Y, Mizutani T, Inamura N, Asano M, Hatori C, Arsmori I, Oku T, Tanaka H.J Med Chem, 2004, 47: 2853

[12] Godfraind T, Miller R, Wibo M. Pharmacol Rev, 1986, 38: 321

[13] Janis R A, Silver P J, Triggle D J. Adv Drug Res, 1987, 16: 309

[14] Mager P P, Coburn R A, Solo A J, Triggle D J, Rothe H. Drug Des Discov, 1992, 8: 273

[15] Mannhold R, Jablonka B, Voigt W, Schoenafinger K, Schraven E. Eur J Med Chem, 1992, 27: 229

[16] Gaudio A C, Korokovas A, Takahata Y.J Pharm Sci, 1994, 83: 1110

[17] Meyer H, Rossert F, Wehinger E, Stoepel K, Vater W. Arzneirn-Forsch, 1981, 31-1: 407

[18] Wang G W, Xia J J, Miao C B, Wu X L. Bull Chem Soc Jpn, 2006, 3: 454

[19] Zolfigol M A, Safaiee M. Synlett, 2004, 827

[20] Sainani J B, Shah A C, Aray V P. Indian J Chem, 1994, 33B: 526

[21] Sufirez M, Ochoa E, Verdecia Y, Verdecia B, Moran L, Martin N, Quinteiro M, Seoane C, Soto J L, Novoa H, Blaton N, Peters O M. Tetrahedron, 1999, 55: 875

[22] Li M, Zuo Z, Wen L, Wang S. J Comb Chem, 2008, 10: 436

[23] Tu S J, Zhou J F, Deng X, Cai P J, Wang H, Feng J C. Chin J Org Chem, 2001, 21: 313

[24] Surasani R, Kalita D, Dhanunjaya-Rao A V, Yarbagi K, Chandrasek- 


\section{Graphical Abstract}

Chin. J. Catal., 2013, 34: 758-763 doi: 10.1016/S1872-2067(11)60518-4

\section{Synthesis of polyhydroquinoline derivatives via a four-component Hantzsch condensation catalyzed by tin dioxide nanoparticles}

Seyed Mohammad VAHDAT*, Fereshteh CHEKIN, Mehdi HATAMI, Maryam KHAVARPOUR, Saeed BAGHERY, Ziba ROSHAN-KOUHI Islamic Azad University, Iran; Khoramshar University of Marine Science and Technology, Iran; Bu-Ali Sina University, Iran

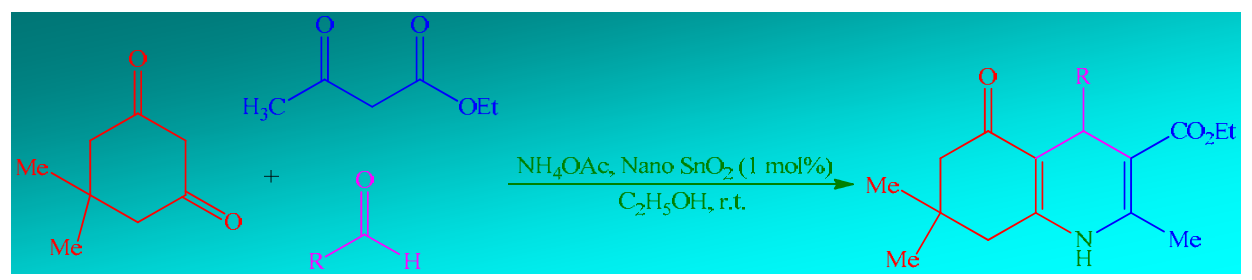

A simple procedure for the $\mathrm{SnO}_{2}$ nanoparticle-catalyzed preparation of polyhydroquinoline derivatives in aqueous ethanol at ambient temperature is described.

har K B. J Fluor Chem, 2012, 135: 91

[25] Saikia L, Dutta D, Dutta D K. Catal Commun, 2012, 19: 1

[26] Sapkal S B, Shelke K F, Shingate B B, Shingare M S. Tetrahedron Lett, 2009, 50: 1754

[27] Maheswara M, Siddaiah V, Damu G L, Venkata-Rao C. Arkivoc, 2006, (2): 201

[28] Tajbakhsh M, Alaee E, Alinezhad H, Khanian M, Jahani F, Khaksar S, Rezaee P, Tajbakhsh M. Chin J Catal (催化学报), 2012, 33: 1517

[29] Kumar A, Maurya R A. Tetrahedron Lett, 2007, 48: 3887

[30] Heravi M M, Bakhtiari K, Javadi N M, Bamoharram F F, Saeedi M, Oskooie H A.J Mol Catal A, 2007, 264: 50

[31] Donelson J L, Gibbs R A, De S K. J Mol Catal A, 2006, 256: 309

[32] Yu Y, Tu M S, Jiang B, Wang Sh L, Tu Sh J. Tetrahedron Lett, 2012, 53: 5071

[33] Cherkupally S R, Mekalan R. Chem Pharm Bull, 2008, 56: 1002

[34] Sabitha G, Reddy G S K, Reddy C S, Yadav J S. Tetrahedron Lett, 2003, 44: 4129

[35] Saha M, Pal A K. Tetrahedron Lett, 2011, 52: 4872

[36] Hong M, Cai Ch, Yi W B. J Fluor Chem, 2010, 131: 111

[37] Reddy C S, Raghu M. Chin Chem Lett, 2008, 19: 775

[38] Adibi H, Samimi H A, Beygzadeh M. Catal Commun, 2007, 8: 2119

[39] Li C J, Chan T H. Organic Reaction in Aqueous Media. New York: Wiley. 1997

[40] Vahdat S M, Baharfar R, Tajbakhsh M, Heydari A, Baghbanian S M, Khaksar S. Tetrahedron Lett, 2008, 49: 6501
[41] Vahdat S M, Khaksar S, Khavarpour M. Chin Chem Lett, 2011, 22: 543

[42] Matloubi-Moghaddam F, Saeidian H, Mirjafary Z, Sadeghi A. J Iran Chem Soc, 2009, 6: 317

[43] Mohammadi-Ziarani Gh, Badiei A R, Khaniania Y, Haddadpour M. Iran J Chem Chem Eng, 2010, 29(2): 1

[44] Karade N N, Budhewara V H, Shindeb S V, Jadhav W N. Lett Org Chem, 2007, 4: 16

[45] Wang L M, Sheng J, Zhang J W, Han J W, Fan Z Y, Tian H, Qian C T. Tetrahedron, 2005, 61: 1539

[46] Ko S, Sastry M N V, Lin C, Yao C F. Tetrahedron Lett, 2005, 46: 5771

[47] Dondoni A, Massi A, Minghini E, Bertolasi V. Tetrahedron, 2004, 60: 2311

[48] Chandrasekhar S, Narsihmulu Ch, Reddy N R K, Sultana S S. Tetrahedron Lett, 2004, 45: 4581

[49] Undale K A, Shaikh T S, Gaikwad D S, Pore D M. Comp Rend Chim, 2011, 14: 511

[50] Brietenbucher J G, Figliozzi G. Tetrahedron Lett, 2000, 41: 4311

[51] Nirmal J P, Dadhaniya P V, Patel M P, Patel R G. Indian J Chem, 2010, 49B: 587

[52] Kumar A, Maurya R A. Synlett, 2008: 883

[53] Sridhar R, Perumal P T. Tetrahedron, 2005, 61: 2465

[54] Reddy B P, Rajesh K, Vijayakumar V. Arab J Chem, 2011, in press, doi:10.1016/j.arabjc.2011.01.027 